\title{
Fernerkundung und Landwirtschaft
}

\section{A. Einleitung}

Landnutzungserhebungen sind von grosser Bedeutung für lokale, regionale, nationale und internationale Entscheidungsgremien. Mit wachsendem Bevölkerungsdruck und steigendem Bedarf an Nahrungsmitteln wird ein verbessertes weltweites Management der landwirtschaftlichen Produkte unerlässlich. Dazu benötigt man zuverlässige Angaben über Anbauort, Qualität und Quantität dieser Produkte. Diese Angaben bilden die Grundlage für landwirtschaftliche Projekte und Entwicklungsprogramme. In jüngster Zeit gelingen mit Hilfe der Fernerkundung sogar relativ zuverlässige Prognosen für die Weizenernte. Dadurch gewinnt diese neue Technologie auf dem Agrarsektor grosse wirtschaftliche und politische Bedeutung und könnte von den Grossmächten sehr wohl als Druckmittel, wenn nicht gar als Waffe eingesetzt werden.

Landwirtschaftliche Bestandesaufnahmen werden von den meisten Ländern der Welt durchgeführt und liefern Informationen über:

- Produktionsflächen (Lage, Ausdehnung)

- Anbauarten (Art und Zustand der Bepflanzung)

- Erträge

- Aussaat- und Erntedaten

- Bodenbeschaffenheit

- Bewässerungssysteme

- Schädlingsbefall

- Katastrophenschäden

- Weideflächen

- Viehbestand

- Zahl und Struktur der landwirtschaftlichen Betriebe

\section{B. Erfassung landwirtschaftlicher Daten}

Landwirtschaftliche Daten werden heute vorwiegend von Regierungen und staatlich unterstützten Organisationen gesammelt. Die FAO (Food and Agricultural Organisation), ein Zweig der Vereinten Nationen , führt alle 10 Jahre einen weltweiten landwirtschaftlichen Zensus (World Census) durch; letztmals also 1970. Neben dem "World Census" werden von der FAO das "Produc- tion and Trade Yearbook", das "Monthly Bulletin of Agricultural Economics" und das "State of Food and Agriculture" herausgegeben.

In den USA werden jährlich Millionen von Dollars für den landwirtschaftlichen Zensus und die statistische Verarbeitung der gesammelten Daten ausgegeben. Als besonders aktuelles Beispiel sei hier das LACIE-Programm (Large Area Crop Inventory Experiment) erwähnt, das in Zusammenarbeit zwischen dem US Department of Agriculture (USDA), der National Oceanic and Atmospheric Administration (NOAA), dem Department of Commerce und der National Aeronautics and Space Administration (NASA) weltweit durchgeführt wird. Bei diesem Programm geht es nicht nur darum, Produktionsflächen $\mathrm{zu}$ erfassen, sondern mit Hilfe von in Satelliten eingebauten Multispektral-Scannern auch die zu erwartenden Erträge vorauszusagen.

Auf Grund der heute zur Verfügung stehenden LANDSA T-2 Daten wurden 1976 in den Test gebieten von Kansas und North Dakota die Weizenerträge mit über $90 \%$ iger Genauigkeit vorausbestimmt. Zudem prophezeiten die USA der Sowjetunion eine ausgezeichnete Weizenernte, was auch eingetroffen ist. Es mag somit nicht erstaunen, dass Russland an LANDSA T-Daten ausserordentlich interessiert ist und diese von den USA auch erhält.

Niemand zweifelt daran, dass sich das heute noch auf Weizen beschränkte LACIE-Projekt auf andere Anbauprodukte wie Reis, Mais etc. ausweiten wird. Der Start der neuen LANDSA T-Satelliten C + D 1978, bzw. 1981, mit besserem Auflösungsvermögen und zusätzlichen Spektralkanälen wird noch genauere Prognosen ermöglichen. Dadurch gewinnt diese neue Technologie auf dem Agrarsektor grosse wirtschaftliche und politische Bedeutung und könnte von den Grossmächten in naher Zukunft sehr wohl als Druckmittel, wenn nicht gar als Waffe eingesetzt werden.

Nüesch Daniel, Dr., Assistent am Geographischen Institut der Universität Zürich, Blümlisalpstrasse 10, 8006 Zürich 
1. Probleme der Datenerfassung Im Zusammenhang mit landwirtschaftlichen Statistiken versucht man immer wieder, Erhebungsmethoden und Datenqualität zu verbessern. Diskutiert werden vor allem die folgenden drei Aspekte:

- Zuverlässigkeit

- Kosten/Nutzen

- Aktualität

Es wird allgemein angenommen, dass die Fernerkundung Möglichkeiten zur Verbesserung bieten kann.

\section{1. Zuverlässigkeit}

Die meisten landwirtschaftlichen Daten werden bis heute mit "konventionellen" Methoden wie Fragebogen, Interviews, Feldbegehungen, usw. gesammelt und fallen daher immer etwas subjektiv aus. In den seltensten Fällen stimmen die Aussagen der Landwirte mit den von Kontrollorganen durchgeführten Tests überein. Diese Diskrepanz kann das Resultat verschiedenster Faktoren sein und verstärkt den Wunsch nach "objektiver" Datenerfassung.

Die Zuverlässigkeit landwirtschaftlicher Bestandesaufnahmen hängt selbstverständlich von den gewählten Stichproben ab. Da eine Stichprobe oder ein Testgebiet nur einen Teil des Ganzen darstellt, sind Verfälschungen wahrscheinlich. Wollte man solche Verfälschưngen vermeiden, müssten die Stichproben oder Testgebiete grösser gewählt werden. Dies ist bei den heute zur Verfügung stehenden Mitteln leider nicht möglich, weil eine vollständige Bestandesaufnahme am Boden viel zu teuer wäre.

\subsection{Kosten}

Der finanzielle Aufwand für eine weltweite Landnutzungserhebung lässt sich kaum abschätzen. Als Anhaltspunkt mag die von den USA für solche Erhebungen ausgegebene Summe von jährlich 50 Millionen Dollars dienen. Diese hohen Kosten rühren davon her, dass ein riesiger Aufwand an Zeit und Arbeitskräften nötig ist für die Planung, die Datensammlung, -verarbeitung und -verbreitung.
1.3. Aktualität der Daten

Der Erfolg landwirtschaftlicher Projekte und Entwicklungsprogramme ist weitgehend abhängig von der Information, die den Entscheidungsgremien zur Verfügung steht. Leider ist es bei den heute gängigen Methoden noch so, dass, da zwischen dem Erfassen der Daten und deren Verfügbarkeit zuviel Zeit verstreicht, in der Planung mit.z.T. überholten Daten gearbeitet werden muss. Tatsächlich nützen einem Landwirt Meldungen über Rostvorkommen in einem Weizenfeld wenig, wenn sie erst kurz vor oder gar nach der Ernte eintreffen. Um eine optimale Verbesserung der Entscheidungsgrundlagen zu erzielen, müssen die Daten also zu einem günstigen Zeitpunkt verfügbar sein.

C. Anwendungsmöglichkeiten der Fernerkundung für landwirtschaftliche Bestandesaufnahmen

Zur Lösung bestimmter landwirtschaftlicher Probleme haben sich Fernerkundungstechniken bereits mehrfach bewährt und werden in Zukunft noch an Bedeutung gewinnen. Das zur Zeit kühnste Projekt auf diesem Gebiet ist das unter B erwähnte LACIE-Programm. Geplant ist zudem die Lokalisierung und Ueberwachung der Opiumanbaugebiete durch $\mathrm{Sa}$ telliten im Rahmen des internationalen Kampfes gegen das Rauschgift. Im weiteren sollen mittels Fernerkundung die Bodenfeuchtigkeit sowie die physiologischen "Stressfaktoren" der Nutzpflanzen erfasst werden. Ziel dieser Erhebung ist u.a., dass bei Wassermangel die Bewässerungsanlagen automatisch einschalten. Obwohl das leicht utopisch klingen mag, besteht durchaus die Möglichkeit, dieses Ziel in den grossen Anbaugebieten zu verwirklichen.

1. Vorteile der Fernerkundung

1.1. Geländebedeckung

Der Einsatz von in grosser Höhe operierenden Sensorträgern ermöglicht es heute, Gebiete, für die früher ein Photomosaik erforderlich war, in einer einzigen Aufnahme zu erfassen. Satellitenaufnahmen erlauben es, ein Gebiet von mehreren Tausend Quadratkilo- 
metern abzudecken. Solche Bilder gewähren dem Interpreten nicht nur einen phantastischen Ueberblick, sie machen eine Landnutzungserhebung in schwer zugänglichen Gebieten überhaupt erst möglich.

\subsection{Zuverlässige Arbeitsgrundlagen}

Jede Satellitenaufnahme ist ein bleibendes Dokument über den jeweiligen Zustand der Landschaft. Im Vergleich mit früheren oder späteren Aufnahmen können Veränderungen in der Landwirtschaft festgestellt, überwacht, ausgewertet und beurteilt werden.

Luftaufnahmen dienen unter Umständen auch als Kartengrundlage und erlauben nach gewissen geometrischen Korrekturen ein genaues Vermessen der Produktionsflächen.

\subsection{Kostenersparnis}

Durch die Verwendung von Luftaufnahmen und mit Hilfe geübter Interpreten lässt sich die Kartenherstellung bedeutend beschleunigen. Einst unerlässliche kostspielige Bodenkontrollen an Ort und Stelle können auf ein Minimum beschränkt werden.

\subsection{Verfügbarkeit des Datenmaterials}

Die Tatsache, dass Luftbilder in relativ kurzer Zeit aufgenommen und ausgewertet werden können, entschädigt für das häufige Fehlen von aktuellem Bildmaterial.

\section{Automatisierungsmöglichkeiten}

Die automatische Auswertung der Fernerkundungsdaten liegt im Bereich des Möglichen und könnte die Verarbeitungszeit wesentlich verringern. Bei der automatischen Auswertung würde die Datenmenge schneller reduziert; dadurch liessen sich die Datensammlung erweitern und die statistische Zuverlässigkeit verbessern.

An dieser Stelle muss aber deutlich gesagt sein, dass die oben aufgeführten Vorteile noch nicht in allen Punkten experimentell oder operationell erprobt worden sind. Trotzdem wird an verschiedenen Instituten fieberhaft an einer Verbesserung dieser Technologie gearbeitet.
3. Grenzen der Fernerkundungstechniken für landwirtschaftliche Bestandesaufnahmen

Eine ganze Reihe von Faktoren, bedingt durch den raschen technologischen Fortschritt, schränken den Einsatz von Fernerkundungstechniken für Landnutzungserhebungen ein. Es bestehen sowohl Hoffnung als auch Zweifel, dass diese Schwierigkeiten in naher Zukunft beseitigt werden können.

\subsection{Mangel an Bildmaterial}

Während einige Industrieländer mit z.T. periodischen Ueberfliegungen eine vollständige photographische Ueberdeckung ihres Gebietes erreichen, verfügen die Entwicklungsländer über kein oder meist veraltetes, nicht flächendeckendes Bildmaterial. Hinzu kommt, dass die Luftbilder aus Kostengründen meist kleinmassstäbig sind und ihr Gebrauch durch militärische Stellen zensuriert, für zivile Zwecke also entweder verboten oder stark eingeschränkt ist.

\subsection{Vielfalt der agrarischen Nutzung} Es ist praktisch unmöglich, einen für die ganze Welt standardisierten Auswertungsprozess einzuführen, der der Vielfalt der agrarischen Landschaft, den landwirtschaft lichen Gewohnheiten, den Anbaupraktiken, den jahreszeitlichen Unterschieden sowie den klimatischen Schwankungen Rechnung trägt. Schon für einen relativ eng begrenzten Raum ist es schwierig, zuverlässige Modelle zu bilden.

\subsection{Fehlende Bodenkontrollen}

Um ein Luftbild möglichst genau interpretieren zu können, sind Bodenkontrollen, wenn möglich während des Ueberfluges unerlässlich. Zur Zeit sind die Auswirkungen verschiedener Parameter wie Richtung und Abstand der Saatreihen, Bodenbeschaffenheit, Bodentemperatur, Bodenfeu chtigkeit, Blattflächenindex (Verhältnis von Blattfläche zu Bodenfläche), Beleuchtungs - und Beobachtungswinkel, usw. auf den Bildinhalt noch nicht vollumfänglich erforscht. Trotz einer beachtlichen Zahl von Bodenkontrollmessungen, wird die Grundlagenforschung noch etliche Probleme zu lösen haben. 


\section{4. Kosten}

Heute noch werden derartige Erhebungen mehrheitlich von Forschungsinstituten geplant und durchgeführt, so dass genaue Kosten-Nutzen-Analysen fehlen. Während über die Kosten solcher Erhebungen bereitwillig Auskunft gegeben wird, gehen die Ansichten über deren Nutzen weit auseinander.

\subsection{Einsatz menschlicher Arbeitskräfte} In vielen Entwicklungsländern ist infolge eines niedrigen Lohnniveaus und eines Ueberangebotes an Arbeitskräften die konventionelle Art der Datenerfassung ökonomischer als teure Fernerkundungsmethoden. Dies gilt vor allem dann, wenn nicht ein Höchstmass an Genauigkeit erforderlich ist.

D. Für landwirtschaftliche Bestandesaufnahmen geeignete Fernerkundungstechniken

Neben der Berücksichtigung der Vor- und Nachteile sind es vor allem zwei Faktoren, die bei der Wahl der Fernerkundungsmethode ausschlaggebend sind:

- die Art der agrarischen Landschaft,

- die Art der verwendeten Fernerkundungstechnik.

Erfassung und Identifikation landwirtschaftlicher Phänomene gehen von der Annahme aus, dass die einzelnen Erscheinungen (z. B. Nutzpflanzen, Bodenbes chaffenheit, usw.) ein durchwegs identifizierbares und differenzierbares Spektralverhalten aufweisen. Parameter, die das unterschiedliche Spektralverhalten bestimmen, sind z. B. : Pflanzenart, Reifegrad, Pflanzenhöhe, Anbaudichte, Unkrautanteil, Bodentemperatur, Bodentyp, Bodenfeu chtigkeit, Bodenbeschaffenheit, usw. Gelingt es dem Interpreten, ein bestimmtes Spektralverhalten mit einem oder mehreren solcher Parametern in Verbindung zu setzen, so wird ihm auch die Identifikation gelingen.

\section{Wahl des Sensors}

Entscheidend für den Erfolg oder Misserfolg eines Bildfluges ist die Wahl des Sensorsystems. Der Sensor soll sowohl bezüglich Bodenauflösung und Bildmassstab als auch bezüglich Kosten optimale Leistungen erbringen.

\section{1. Photographische Sensoren}

Obwohl sich der sichtbare Spektralbereich im Vergleich zum gesamten Spektrum unver hältnismässig klein ausnimmt, bleibt er vorläufig der informationsreichste Teil der bisher erschlossenen Spektralbereiche. Die traditionelle Luftphotographie, die sich vor allem der sichtbaren und nahinfraroten Strahlung bedient, hat heute ihre Bedeutung keineswegs verloren, doch ist man an Grenzen gestossen, die aus physikalischen und technischen Gründen (Filmhaltbarkeit, Filmemulsion, Optik, usw. ) nicht überwunden werden können.

Einige Vorteile der photographischen Aufnahme seien hier kurz erwähnt:

- metrische Bildqualität, d.h. die Bilder lassen sich korrigieren, so dass genaue Messungen gemacht werden können,

- hohes Auflösungsvermögen,

- Möglichkeit der stereoskopischen Betrachtung,

- Möglichkeit von Film/Filterkombinationen,

- relativ geringe Kosten,

- grosse Aehnlichkeit des Bildinhaltes mit der Situation am Boden, was die Interpretation erleichtert,

- gute Interpretationsmöglichkeiten durch Menschen oder Maschinen.

Da Schwarz-Weiss-Filme oft nur beschränkte Wiedergabemöglichkeiten bieten, benützt man immer häufiger die teureren Farbund Falschfarbenfilme, die erwiesenermassen den potentiellen Informationsgehalt der $\mathrm{Bil}$ der zu steigern vermögen. Die Verwendung von Falschfarbenfilmen, die für nahinfrarote Strahlung empfindlich sind, hat sich vor allem zur Erfassung von Vegetationsschäden bewährt.

\subsection{Multispektral-Scanner}

Die in Flugzeugen und Satelliten eingebauten Multispektral-Scanner (dt. : Mehrkanal-Abtastgeräte) eröffneten den Erdwissenschaften ganz neue Möglichkeiten zur besseren Erfassung der Erdoberfläche. Für Landnutzungserhebungen bietet der Einsatz des Multispektral-Scanners folgende Vorteile: 
- simultane Datenregistrierung in mehreren Spektralbereichen (Kanälen), auch im nicht sichtbaren Bereich,

- Möglichkeit der elektronischen Datenübermittlung an Bodenstationen,

- simultane Darstellung der registrierten Daten auf einem Bildschirm,

- geringere Kosten bei Satelliten-Aufnahmen, insbesondere bei periodischen Ueberfliegungen.

Daneben sollen jedoch die Nachteile nicht unerwähnt bleiben:

- geringeres Auflösungsvermögen am Boden,

- Bildverzerrungen, vor allem bei in Flugzeugen eingebauten Instrumenten,

- kostspielige und komplizierte Aufnahmeund Wiedergabesysteme, die ein geschultes Fachpersonal nötig machen.

Trotz aller Nachteile werden MultispektralAufnahmen dank verbesserten Aufnahmeund Verarbeitungstechniken in naher Zukunft auch für landwirtschaftliche Aufgaben die photographischen Systeme ernsthaft konkurrenzieren.

1.3. Aktive Mikrowellen (SLR, Side-looking radar)

Systeme, die mit aktiven Mikrowellen arbeiten, bieten vor allem für landwirtschaftliche Aufgaben wesentliche Vorteile:

- Einsatz bei Tag und Nacht möglich,

- Einsatz bei nahezu jedem Wetter möglich,

- grosse Geländebedeckung mit vorwiegend einheitlicher Bodenauflösung.

Der Einsatz von SLR hat sich bis heute vor allem bei Boden- und Landnutzungsunter suchungen in tropischen Gebieten bewährt, in denen die Bewölkungssituation eine landesweite Befliegung oft verunmöglicht.

Die Nachteile des SLR sind denen des Multispektral-Scanners ähnlich. Zudem besteht das SLR-Bild aus Signalen, die das System selber aussendet und wieder empfängt. $\mathrm{Zu}$ den Parametern, die das Spektralverhalten der Objekte ohnehin beeinflussen, kommt also noch diese "aktive Beleuchtung", was die Interpretation der wenig vertrauten, nicht konventionellen Bilder sehr erschwert.

\subsection{Passive Mikrowellen}

Aus der Literatur geht hervor, dass passive Mikrowellen wegen der geringen Bodenauflösung für die Landnutzungsinterpretation kaum Verwendung finden. Hingegen bieten sie vielversprechende Möglichkeiten zur Erfassung der Bodenfeuchtigkeit und der Wasserreserven.

\section{Periodische Aufnahmen}

Bedingt durch die dymamische Natur der agrarischen Landschaft ist es wünschbar, oft notwendig, die Daten in bestimmten Interval len zu erfassen. Dazu werden Systeme benötigt, die in möglichst kurzer Zeit möglichst genaue Daten erheben und verarbeiten können. Zur Zeit muss aus finanziellen Gründen auf den periodischen Einsatz von Aufnahmesensoren von Flugzeugen aus verzichtet werden. Gerade hier aber liegen die grossen Möglichkeiten von Satellitensystemen.

\section{E. Stand der Fernerkundungsforschung}

Die heute angewandten Verfahren haben, darüber muss man sich im klaren sein, ihren Ursprung im militärischen Bereich und wurden erst mit der Zeit für zivile Bedürfnisse freigegeben und modifiziert. Ohne die gewaltigen finanziellen Aufwendungen der Grossmächte, hätte eine derart rasante Entwicklung nie stattfinden können. Gleichzeitig haben folgende Tatsachen den raschen Fortschritt begünstigt:

- die Entwicklung von Geräten, die die elektromagnetische Strahlung in verschiedene Wellenlängenbereiche aufspalten,

- die Entwicklung von Geräten, die die für das Auge unsichtbare Strahlung registrieren,

- der Ausbau der digitalen Datenregistrierung und -verarbeitung,

- die Steigerung der Datenübertragungsund Datenspeicherungsrate.

Seit dem Start des ersten zivilen Erdbeobachtungssatelliten ERTS-1 (Earth Resources Technology Satellite) im Juli 1972 stehen einem grossen Benutzerkreis Bilder eines 4-Kanal-Multispektral-Scanners zur Verfü- 
gung. Es zeigte sich bald, dass mit den herkömmlichen Methoden die Interpretation dieser Bilder zwar möglich ist, dass wegen der anfallenden Datenmenge aber eine ausgesprochen schlechte Ausnützung des Informationsgehaltes resultiert. Denkt man an die wichtigsten Fernerkundungsprojekte der nächsten Jahre (geostationäre Wettersatelliten, Landsat-3, Spacelab, usw. ) mit Nutzlasten von einigen Tonnen und bis zu 30 Sensoren für Erderkundungsmissionen, versteht man die Forderung nach drastischer Datenreduktion mit minimalen Informationsverlust.

1. Forschungsprojekte

1.1. Spektraldaten

Um die notwendige Feldarbeit auf ein absolutes Minimum beschränken zu können, versucht man einen Schlüssel für alle möglichen Oberflächenbedeckungen zu erstellen, mit dessen Hilfe sich die verschiedensten Untersuchungsgebiete bearbeiten lassen. Man müsste also eine Datenbank unvorstellbaren Ausmasses einrichten, in der die Schwankungen der Spektraldaten innerhalb eines Tages als Funktion der Aufnahmezeit (Sonnenwinkel und Azimut), des wechselnden Beobachtungswinkels sowie der atmosphärischen Bedingungen berücksichtigt sind. In Ermangelung einer solchen Datenbank versucht man, Korrekturmodelle zu finden, mit denen die aufgenommenen Daten berichtigt werden können.

\subsection{Automatische Klassifikation} Vor allem bei der Verwendung multispektraler Abtastgeräte werden die Messungen unmittelbar nach der Registrierung in digitale Form überführt und gespeichert. Diese neue Art der Datensammlung und die Menge der anfallenden Daten erfordern eine automatische Datenverarbeitung. Mit Hilfe verschiedener Entscheidungsverfahren aus der mathematischen Statistik werden die einzelnen Messungen einer entsprechenden, vom Interpreten bestimmten Klasse zugeordnet. Beim heutigen Entwicklungsstand der Klassifikationsverfahren ist es nur noch speziell ausgebildeten Fachkräften möglich, Verbesserungen zu erzielen. Ihnen geht es vor allem darum, eine Datenreduktion mit möglichst geringem Informationsverlust zu erreichen und Algorithmen zu entwickeln, die in kürzerer Rechenzeit ebenso gute oder bessere Resultate liefern. Vorläufig sind einer weiteren Entwicklung der automatischen Klassifikation durch die grosse Abhängigkeit der Merkmalsidentifikation von der spektralen Rückstrahlung Grenzen gesetzt. Für eine weitere Verbesserung der Klassifikationsresultate wird es unumgänglich sein, neue $\mathrm{Pa}$ rameter wie z. B. die Textur in die Algorithmen miteinzubeziehen.

\section{F. Schlussbemerkungen}

Die Annahme, Fernerkundungsmethoden spielten für künftige landwirtschaftliche Bestandesaufnahmen eine immer wichtigere Rolle, geht davon aus,

- dass die konventionelle Datensammlung durch menschliche Arbeitskräfte eher ungenau und $\mathrm{zu}$ teuer ist,

- dass Unzulänglichkeiten wie z. B. mangelnde Aktualität der Daten, Unzugänglichkeit landwirtschaftlicher Gebiete in Entwicklungsländern, usw., durch Fernerkundungsmethoden eliminiert werden können,

- dass neue, in der Forschung erfolgversprechende Methoden wie der Einsatz neuer Sensoren und Sensorträger, periodische Aufnahmen eines Gebietes, neuartige Datenerfassungstechniken und Datenverarbeitungstechniken, usw., sich auch in der Praxis durchsetzen,

- dass mit Fernerkundungsmethoden Verbesserungen und Fortschritte erzielt werden können, die der Landwirtschaft in absehbarer Zeit direkten oder indirekten Gewinn einbringen.

Die grosse Anzahl der bis heute auf der ganzen Welt durchgeführten Bildflüge deutet darauf hin, dass die nicht kostendeckenden Missionen durch andere, erfolgreiche aufgewogen werden. Auch wenn der Erfolg nicht unmittelbar offenkundig ist, sind bei verschiedenen Bewässerungsprojekten, Schädlingsbekämpfungsaktionen und Inventuren langfristig doch beträchtliche Einsparungen erzielt worden. 
Thamann R. (1974) erwähnt Zahlen von Luney und Dill für die Vereinigte Arabische Republik, in der jährliche Verluste von 168 Millionen Dollars durch Pflanzenkrankheiten und Insektenbefall bei den wichtigsten Getreidesorten zu verzeichnen sind. Im weiteren zitiert er Schätzungen von Hoffer, wonach die USA für Unkrautbekämpfung jährlich 3.8 Milliarden Dollars ausgeben. Wenn sich diese enormen Summen durch Fernerkundungsmethoden nur um einige Prozente verringern liessen, wären die dafür nötigen Beträge schon gerechtfertigt.

Selbstverständlich bestehen für Fernerkundungsmethoden noch weitere Anwendungsmöglichkeiten. Eine der dringendsten und erfolgversprechendsten Aufgaben auf dem Agrarsektor sind Landnutzungskartierungen in Entwicklungsländern, wo diesbezüglich praktisch keine Informationen zur Verfügung stehen. Vor allem die von Satelliten aus betriebene Fernerkundung wird Entscheidungsgrundlagen beschaffen und offene Fragen klären helfen.

\section{LITERA TUR VERZEICHNIS}

AVERY E., 1977: Interpretation of Aerial Photographs. Burgess Publishing Company, Minneapolis.

BARETT E., CURTIS L., 1974: Environmental Remote Sensing - Applications and Achievements. Edward Arnold, London.

BINZEGGER R., 1975: ERTS-Multispektraldaten als Informationsquelle für thematische Kartiemungen. Diss. Univ. Zürich.
COLWELL R. N., 1969: A Summary of the Uses and Limitations of Multispectral Remote Sensing. Proc. IRSI (Int. Remote Sensing Institute), Vol. II, Sa cramento, California.

COLWELL R. N., 1972: Applications of Remote Sensing in Agriculture and Forestry. Proc. Int. Workshop on Earth Resources Survey Systems, Vol. I, Washington.

DUCROCQ A., 1977: Les musses et le programme LACIE. Air et Cosmos, $N^{\circ} 655$.

NUEESCH D., 1977: Landnutzungsinterpretation mit Hilfe multispektraler Abtastverfahren. Diss. Univ. Zürich.

PLESSEY RADAR, 1973: Multispectral Scanning Systems and their Potential Application to Earth Resource Surveys. Prep. under ESRO Contract No ESTEC 1673/72 EL, Vol. 4: Earth Science Applications.

THAMANN R., 1974: Remote Sensing of Agricultural Resources. in: Remote Sensing, Techniques for Environmental Analysis, Hamilton Publishing Company, Santa Barbara Calif.

TRACHSLER H., 1974: Luftbild und Orthophoto als Datenquelle für geographische Informationssysteme; dargestellt am Beispiel einer gesamtschweizerischen Landnutzungserhebung. Diss. Univ. Züri ch.

WENDEROTH S. et al, 1974: Multispectral Photography for Earth Resources. University of Long Island, Greenvale NY. 